\title{
Pedestrian Mobility in Theme Park Disasters
}

\author{
Gürkan Solmaz and Damla Turgut \\ Department of Electrical Engineering and Computer Science \\ University of Central Florida \\ Email: \{gsolmaz,turgut\}@eecs.ucf.edu
}

\begin{abstract}
Realistic mobility simulation is critical for evaluating the performance of communication networks. Although various mobility models exist, they do not capture the changes in the mobility decisions of pedestrians in specific environments. For instance, in the case of a natural or man-made disaster, the main goal is the safe evacuation of the area, creating unique pedestrian mobility patterns.

In this paper, we focus on the scenario of evacuating a theme park in response to a disaster. We discuss the characteristics of theme parks, modeling the environment, and the mobility decisions of pedestrians. Real theme park maps are used for modeling the environment with roads, physical obstacles, and simulating disaster events. The mobility decisions of the pedestrians are based on the evacuation goal, the limited knowledge of the area, and the obstacles. The impact of the interactions between the crowd flows is modeled based on the concept of social force. The model is evaluated by comparison with the existing mobility models and the GPS traces of theme park visitors.
\end{abstract}

\section{INTRODUCTION}

Theme parks are large and crowded areas with thousands of daily visitors. Particularly, large-scale theme parks attract visitors from all over the world. In areas such as Central Florida, which hosts five of the top ten theme parks with highest attendances in the world [1], the theme parks can represent a significant part of the region's economy. At the same time, the region also has a history of natural disasters including hurricanes, tornadoes, and tropical storms.

Managing the flow of visitors in a theme park is a significant challenge due to the high volume of visitors, as well as the large area and many physical obstacles present in the park. These challenges become even more acute in the event of a natural or man-made disaster, when a large number of visitors must be securely evacuated while performing targeted search and rescue missions at various locations in the park. To coordinate these activities, the operators of theme parks require a robust wireless communication system. As the services based on a pre-installed infrastructure might be disrupted in the case of a disaster, many recent studies considered the use of more resilient, infrastructure-independent systems. Examples of such systems include communication systems that includes smartphones and other mobile devices as opportunistic communication networks [2] and wireless sensor networks with mobile sinks [3].

As these systems use network nodes whose mobility follows that of their human owners, the overall performance is dependent on the mobility of the participating humans. Thus, to evaluate the performance of the communication network, we need a realistic simulation of the human mobility in the specific scenario and environment. Commonly used mobility models such as random waypoint or generic human mobility models do not approximate well human movement in theme parks, which is characterized by a highly structured, usually purposeful movement dependent on the environment. In [4], we modeled the human mobility of theme park visitors, assuming an usual day of visitors exploring the attractions of the park. In case of a disaster scenario, however, the behavior and goals of the visitors change: they try to avoid areas impacted by the disaster, find easy ways to secure places, and escape from the disaster areas on the fastest possible route [5].

In this paper, we present a mobility model of the pedestrians in theme park disasters (TP-D). We use real theme park maps to model the disaster areas that include physical obstacles, roads, and exit gates. The macro and micro mobility behaviors of the pedestrians are modeled with the theme park model and the social force concept that represents the crowd flow dynamics by social interactions. For the evaluation of TP-D, we capture the network characteristics and analyze diffusion of the mobile nodes. We analyze the model and compare its outcomes with existing mobility models and real life mobility traces of theme park visitors. Our model provides a realistic representation of the human mobility and it can be used as a baseline for network simulations and the testing of disaster management strategies.

\section{Mobility Model}

\section{A. Characteristics of Theme Parks}

Before starting to describe the model in detail, let us first explain the unique characteristics of theme parks by a mobility modeling perspective. Theme parks are very large but bounded entertainment areas. The area of a theme park includes attractions and roads. We use the term attraction to denote the places in which people gather and spend time. Rides, restaurants and live events are classified as the attractions. Roads are the pedestrian ways which connect the attractions to each other and to the exit gates. Vehicles have limited use inside theme park areas, while exit gates are usually located close to parking lots.

Theme parks are open-air areas but they also have buildings such as indoor rides, restaurants and gift shops. The area of theme parks include many man-made and natural obstacles for pedestrians. People who spend their day in theme parks have activities such as visiting rides, walking among the attractions along the roads, and eating at the restaurants.

Due to the nature of the large and crowded area, a natural or man-made disaster may have devastating effects on a theme 


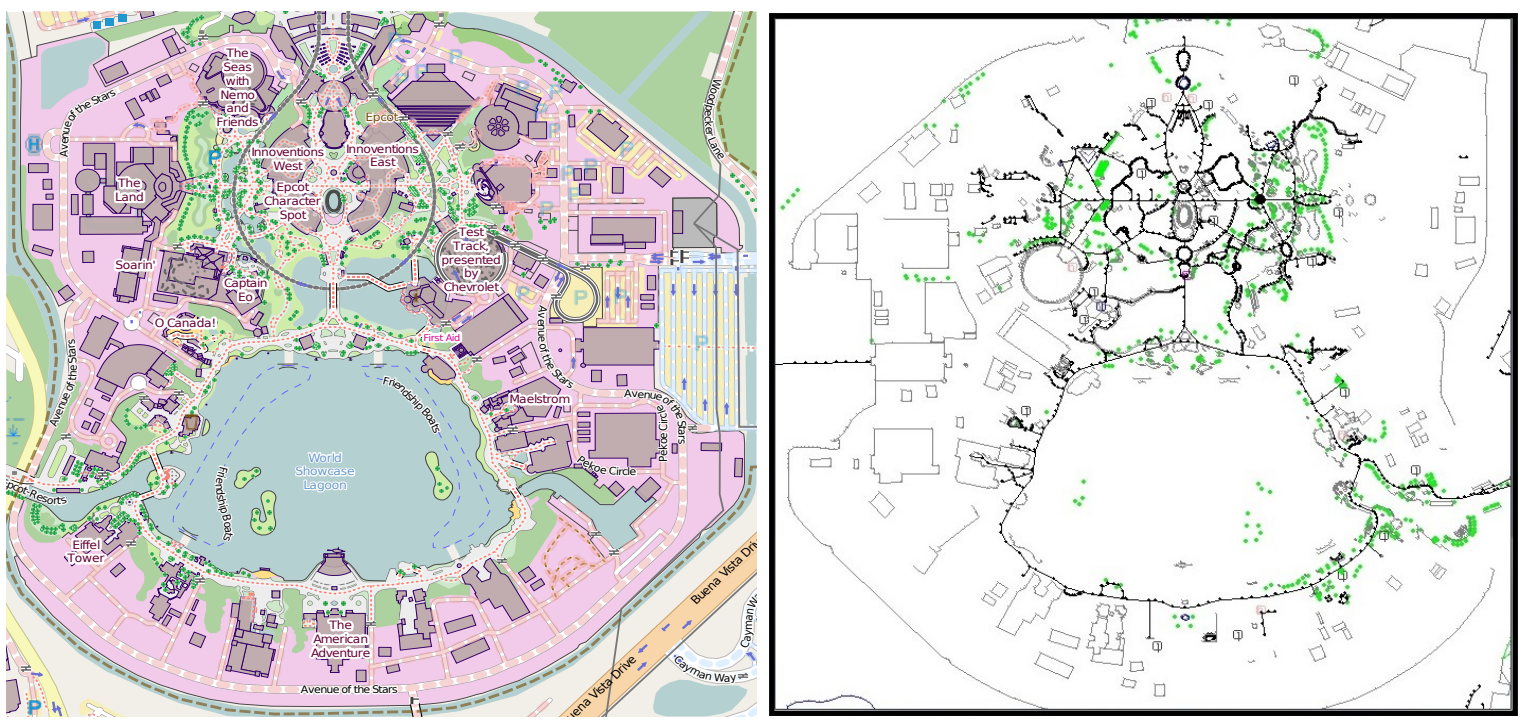

Fig. 1. The maps of the Epcot park. Left: the map extracted from (OSM), right: the processed map with 1655 waypoints.

park. As a disaster response strategy, in time of a disaster, the main goal is secure and fast evacuation of the visitors. Considering an example of tornado alert in a crowded day, the visitors should leave the park to reach the transportation services located outside the park. Since there are thousands of people leaving the park, the mobility of a single pedestrian cannot be considered independently from the others. As a result, social interactions between the pedestrians that may cause slowdowns in pedestrian flows, should be considered for realistic mobility modeling.

The evacuation problem of theme parks is different from other evacuation problems. For instance, in a city scenario, the main purpose is fast evacuation of the city by the effective share of streets by cars and public transportation services. Other types of evacuation scenarios focus on indoor evacuation, such as evacuation from buildings or from rooms of a building. The evacuation problem of theme parks includes large areas with physical obstacles and high numbers of pedestrians. As expected, the movement of pedestrians during disasters has many differences compared with their movement in ordinary times. Because of the aforementioned characteristics, theme parks require scenario-specific mobility modeling for performance evaluation of networks as well as simulating and testing various evacuation strategies.

\section{B. Modeling Theme Parks}

We model the theme park as the combination of roads, obstacles, lands, and disaster events. Each road contains a set of waypoints, which are the movement points for the theme park visitors. In this case, the length of a road is equal to the sum of the distances between each pair of its consecutive waypoints. The roads show the possible ways to reach the target locations in the map. The gates are considered as the target locations and they are placed close to the borders of the park as they connect the theme park with the outside world.

Attractions are composed of man-made buildings and other structures. In the ordinary times, the main goal of the visitors is to visit the attractions. For a disaster case, when the visitors should be evacuated from the disaster area as quickly as possible, we consider these buildings as obstacles which prevent the free flow of the visitors. Furthermore, we model the other man-made structures in the park such as fences and walls as obstacles. There are also natural obstacles such as lakes, trees, forest, river, and so on.

The areas which neither include the obstacles nor the roads are classified as the lands. The lands can be used by pedestrians but they are not preferred unless there are unexpected conditions on the available roads. For instance, when a road is unavailable due to an impact of the disaster event, the lands might be used instead. In some cases, lands provide shortcuts between the waypoints. Disaster areas are classified as the redzones and they are the circular areas reflecting the effects of the disaster. In a real scenario, one can think the red-zones as the events which damage roads or bridges, caused by an earth-quake, a hurricane, a fire, a terrorist attack, and so on. The red-zones have radius values which specify the damaged areas and active times. If a red-zone is in its active time and it effects an area including some portions of a road, the road is assumed to be unavailable at that particular time.

The model of the theme park can be created synthetically or using real maps. We use OpenStreetMap (OSM) [6] to extract the real theme park maps and parse the OSM data to generate the roads, the obstacles, the lands, and the gates. We collect the waypoints using the OSM data and connect the consecutive waypoints to create the roads. Roads have width values according to their OSM types (footway, path, and pedestrian way). Fig. 1 displays an example of the real map of Epcot from Walt Disney World in Orlando (left-side), and the processed version of the map including the waypoints, the roads, the gates, and the obstacles (right-side). In this figure, the small black dots represent the waypoints, while the black lines connecting the waypoints are the roads and the closed polygons are the obstacles. The model also includes red-zones which are added to the model according to their active times; 


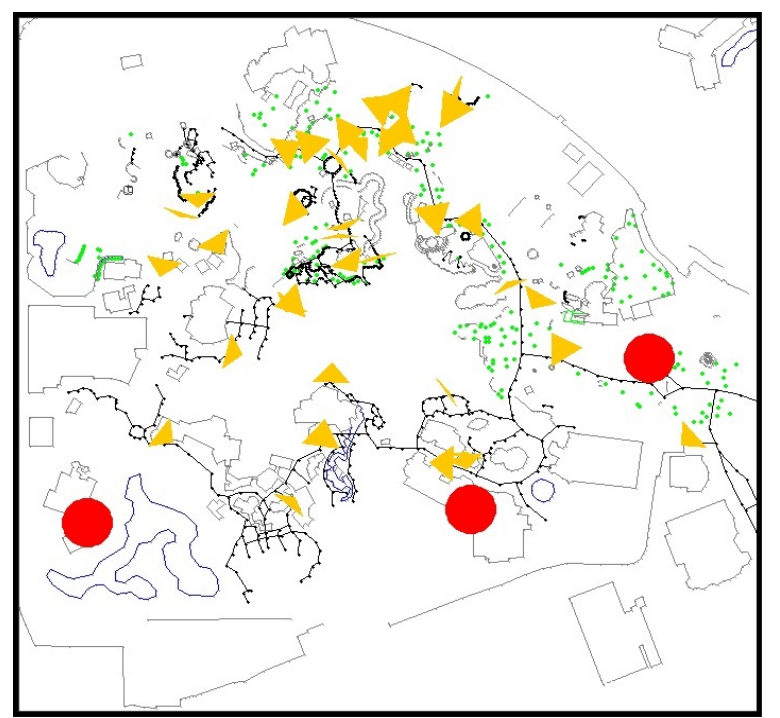

Fig. 2. Islands of Adventure theme park model: 700 waypoints, 40 pedestrians and 3 red-zones.

however, they are not included in this initial processing of the map. While theme park models are generated computationally, it is possible to create a non-existing theme park in design stage manually and generate a model in the same fashion.

\section{Mobility Model of Emergency Evacuation}

We describe the mobility behavior of the visitors as follows. Initially, the visitors are randomly positioned to one of the waypoints in the theme park model. Each visitor selects an exit gate among the available exit gates in the park and marks its position as the target point. A visitor is assumed to be evacuated after reaching one of the exit gates. The visitor tries to reach the target point by moving among the waypoints. Whenever the visitor reaches a waypoint, the waypoint is marked as visited. The next destination point is selected among all the visible waypoints. The visited waypoints, the waypoints positioned in a red-zone or the waypoints which are not visible to the visitor are not taken into consideration as the candidates for the next destination point. The visitor selects a new waypoint according to its distance and direction from the current position since the visitor tries to select the destination point closer to the target. The selection of the waypoints is constrained by the the visitor's knowledge about the world, the obstacles, and possible active red-zones along the way. If a visitor cannot find any waypoint as a candidate for the next destination, the new destination is selected by exploration with a random direction. The random exploration distance is a parameter which bounds the flexibility of the movement of the visitors in cases of the unexpected disaster events. Another parameter which effects the flexibility the most is the visibility parameter. The visibility may differ according to the type and the impact of the disaster. We classify all the above steps of a visitor considering the global movement starting from the initial point to the target point as the macro-mobility behavior of the visitors.

The visitors have the local knowledge of their environment and they know the gate from which they entered the park.
The local knowledge of the visitors is determined by the visibility parameter that specifies the visible distance for each visitor and the obstacles which may be located along the way. The visibility parameter represents the radius of the circular visible area. The visibility can have a constant or variable value according to different disaster scenarios. We mostly use a fixed value for simulations, because it basically represents the impact of the weather conditions such as heavy mist in a relatively short period of evacuation time. This model aims to serve as a baseline for testing disaster response methods. The movement of the pedestrians are modeled as they are on their own, without any help by communication devices or theme park operators for the evacuation of the pedestrians. The visitors are not assumed to communicate with each other and there is no broadcasting system for raising the awareness.

The speeds of the visitors differ from each other. Each visitor has a maximum speed which depends on the physical attributes of the individual such as age, gender, and weight. The speed of each individual is determined randomly between a global minimum and a global maximum speed of the visitors. The speed of a visitor varies from 0 to the local maximum speed. The local maximum speed is the speed when the visitor is free to walk without disturbance or the obstacles. In the disaster scenario, the actual speed of a visitor is less than the local maximum speed most of the times due to the effects of the social interactions.

We consider micro-mobility as the mobility of a visitor between the two consecutive waypoints separately from the macro-mobility model and the theme park model. We use the social force model (SFM) [7] which is a mathematical model of pedestrian flows sharing the same roads and used by various simulators of human mobility. According to the social force concept, the behavioral changes in the human motion are actually caused by the combination of the social interactions. We model the social forces on the visitors according to their social interactions with each other using SFM. By this model, the visitors adapt their speed and direction of the movement from a waypoint to another. Please refer to [5] for the details on how we used SFM in our application scenario.

The main effect of SFM in the theme park scenario is that the usage of the same roads by the visitors causes an increase in the social interactions. This increase slows down the flow of the visitors along the roads. Since the theme parks are crowded areas with pedestrian ways, we believe that the social force model is the best-fit model to represent the crowd dynamics and the micro-mobility behavior for the evacuation of the visitors from the theme parks.

Fig. 2 illustrates the complete theme park model generated using the map of the Universal's Islands of Adventures park and the inclusion of the visitors and the red-zones. Forty visitors moving along the roads are represented by the yellow triangles. The shape of the triangles illustrate the directions and velocities of each of the visitors. Three red-zones are demonstrated by the big red circles. 


\section{Model Evaluation}

\section{A. Simulation setup and metrics}

The mobility metrics can be classified in three types: movement-based, link-based, and network-based metrics. The movement-based metrics are usually extracted from analyzing individuals' movement patterns. Flight lengths, average velocity, waiting times, mean-square distances are among the movement-based metrics. The link-based metrics focus on the overall picture of the area and analyze the effects of the mobility with respect to the relations between the mobile nodes. Average node density, variance of node density, average pairwise distances, relative mobility are examples of the link-based metrics. The network performance-based metrics are used for analyzing the effects of the mobility on the performance of the networks. In [5], we analyzed the movement-based results and evacuation times while in this study we evaluate the link-based and network performancebased results.

The simulation of TP-D generates the mobility traces of pedestrians. We evaluate the characteristics of the resulting traces and compare them with existing synthetic mobility models as well as the GPS traces of theme park visitors. The simulations of the theme park mobility model (TP) [4], self-similar least action walk model (SLAW) [8], and Random Waypoint Model (RWP) are conducted. TP model specifically considers realistic mobility behaviors of the theme park visitors, while SLAW is a more generic human mobility model. We also include RWP since it is the most commonly used model in network simulations. The GPS traces, provided by CRAWDAD archive, are collected from theme park visitors who spent their holidays at Walt Disney World. The traces are processed to filter out the times of traveling in a vehicle between the four Disney parks.

The terrain size of the disaster simulation area depends on the size of the modeled park. We use the map of Magic Kingdom in Orlando since it is the most popular theme park in the world. To model the social interactions, the circular specification of SFM is used with angular dependencies and empirical values, proposed by Helbing and Johansson [7]. Let us now summarize the simulation parameters. We have 2000 pedestrians. The simulation time is $2000 \mathrm{~s}$ with $0.5 \mathrm{~s}$ sampling time. We assumed a fixed visibility of $100 \mathrm{~m}$ for pedestrians with $1.0 \mathrm{~m} / \mathrm{s}$ maximum speed and $0.0 \mathrm{~m} / \mathrm{s}$ as the minimum speed. Fifty red-zones are generated randomly with $100 \mathrm{~m}$ radius and $1000 \mathrm{~s}$ of active times. The random movement distance of pedestrians is considered $10 \mathrm{~m}$. For SFM, the interaction strength is $0.11 \pm 0.06$ with range $0.84 \pm 0.63$. The relaxation time $(\tau)$ and the lambda $(\lambda)$ values are set to $0.5 \mathrm{~s}$ and 0.1 respectively.

\section{B. Analysis of the Traces}

Fig. 3 shows a snapshot from the simulation of 2000 pedestrians in the Magic Kingdom park. The pedestrian flows to the exit gates can be seen on the roads.

1) Experiment 1 - Average node degrees: The node degree of a pedestrian is defined as the number of neighbor pedestrians. The neighbors of the pedestrian are considered within

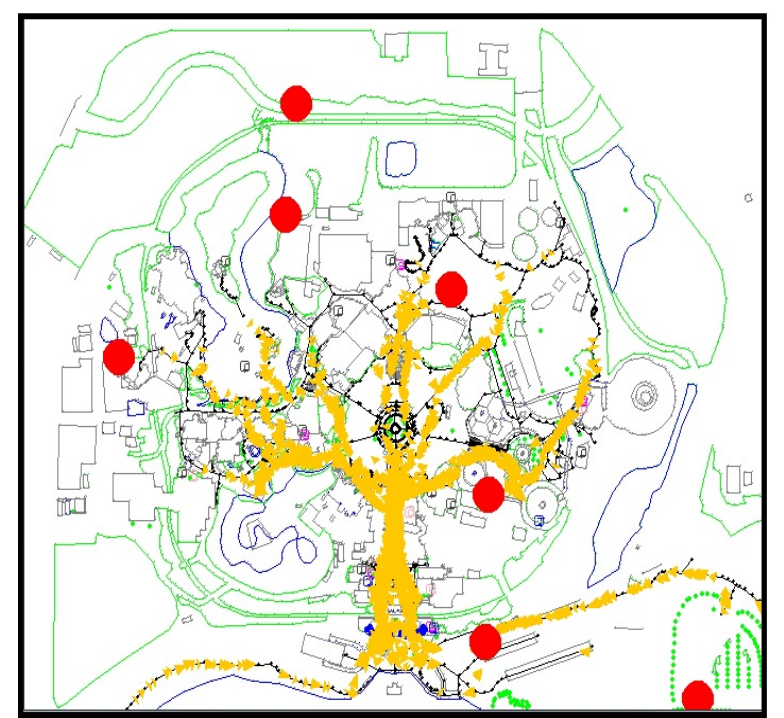

Fig. 3. The crowd flows in Magic Kingdom park and effect of the red-zones.

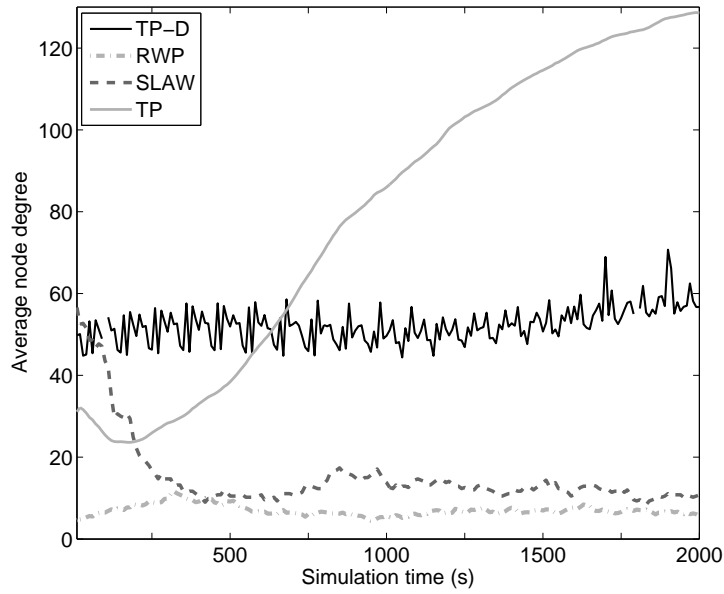

Fig. 4. Average node degrees by varying simulation times for TP-D, TP, SLAW, and RWP models

the communication range of the pedestrian. In other words, two neighbors are assumed to have a wireless communication link between them if they are in the communication range of each other. Average node degree is a link-based metric that is calculated by averaging the node degrees of all the pedestrians. Basically, a higher average node degree yields a better network performance. We assume a transmission range of $40 \mathrm{~m}$ and observe the effects of the pedestrian movements on the average node degree by the varying simulation times. The results are normalized to 1000 visitors in each model.

Fig. 4 shows the average node degrees by the varying simulation times for TP-D, SLAW, TP, and RWP. All the mobility models generated distinct characteristic changes in node degrees with respect to the simulation time. TP-D has the highest average node degree at the initial phase since the mobile nodes are initially distributed only on the roads while other models distribute the visitors to the entire area. We also see that the average node degrees increase steadily for TP-D, 


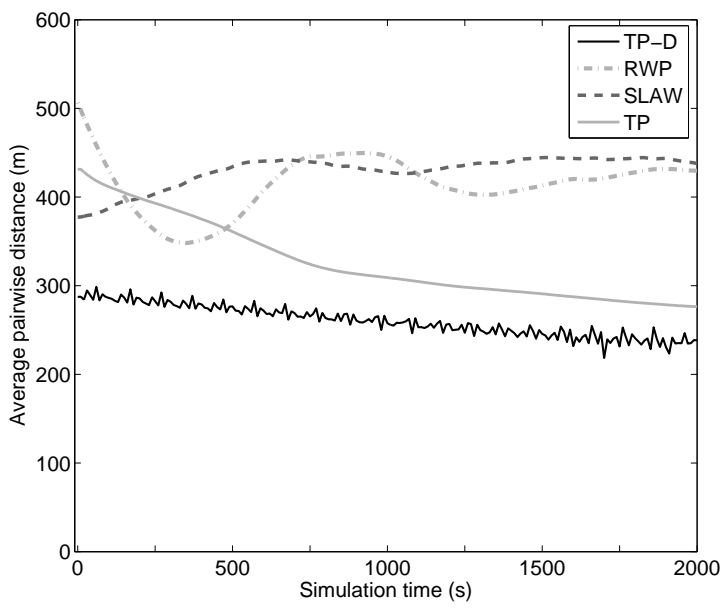

Fig. 5. Average pairwise distances by varying simulation times for TP-D, TP, SLAW, and RWP models.

while the values may vary in short period of times because of the shorter sampling time. The node degrees increase much faster in TP model due to the gathering behavior of visitors in the attractions compared to the TP-D model. In TP, visitors start waiting in the queues very close to each other. As a result, we see higher average node degrees after 2000s. In TP-D, the pedestrians travel along the roads together, which does not produce the effect of the gathering behavior. Because of the slowdowns in pedestrian traffic, the number of people close to exit gates and main roads increase, producing the increase in the node degrees. SLAW model has an initial phase of 500s and the results converge to a constant level. RWP stays constant with some variances in short times caused by the randomness.

2) Experiment 2 - Average pairwise distances: The distances between all pairs of mobile nodes are averaged to calculate average pairwise distances. As a link-based metric, it helps us to evaluate the closeness of a node to another on average and shows the possibility to form a new network with a desired subset of all the mobile nodes. Smaller pairwise distances are expected for better network performance. As in the previous experiment, we observe the effects of the mobility on the results by the varying simulation times.

As it can be seen in Fig. 5, all the models again present different characteristics. TP-D has an overall constant decay of average pairwise distances. As also observed in the previous experiment, the pedestrians become closer to each other as the time moves on. An interesting difference is the fact that the significance of the effect of TP becomes weaker. This is due to the consideration of the entire population for each individual. For instance, when a visitor goes to an attraction, the pairwise distance with the other people in the same attraction becomes smaller, while the visitor's pairwise distance with people in other attractions of the park may become larger. In TP$\mathrm{D}$, on the other hand, the people move to the same target. Furthermore, since we no longer take the pedestrians, who reached the exit gates, into consideration, the fast increase due to the gathering behavior does not occur in TP-D. After

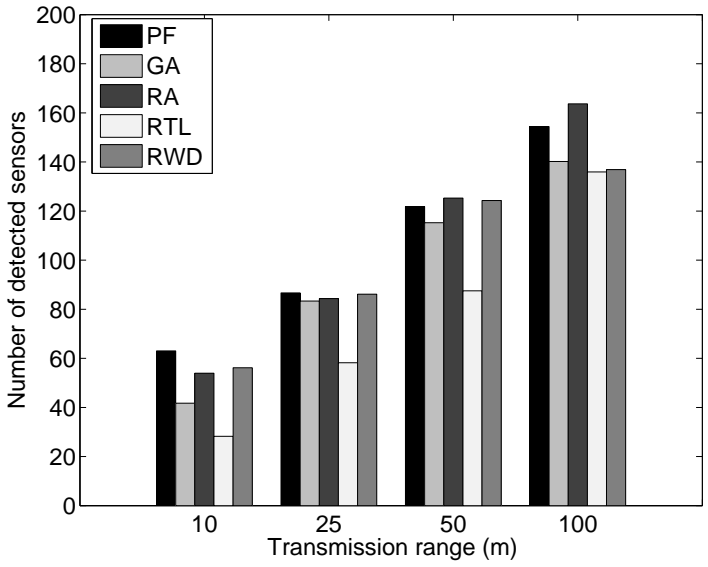

Fig. 6. Number of detected sensor nodes for various mobile sink mobility models with varying transmission ranges.

the initial phase of 500s, SLAW and RWP models have constant average pairwise distances with some variances due to randomness in the models.

3) Experiment 3 - Number of detected sensor nodes: We used the mobility model as a baseline for a simulation of an opportunistic network consisting of 200 sensor nodes that are smart-phones carried by theme park pedestrians and 5 mobile sinks. As a disaster response strategy, mobile sinks are used for search and rescue operations and tracking the pedestrians in the theme park during the evacuation process. In this experiment, the mobile sinks broadcast a message to the sensor nodes using epidemic routing and mark the sensor nodes as detected if they send acknowledgment. We observed the total number of detected sensors and analyzed the effects of various mobile sink movement strategies on the network's coverage performance. As shown in Fig. 6, physical force (PF) and road allocation (RA) based sink mobilities are the overall winners of such scenario reaching up to $80 \%$ of the nodes compared to grid allocation (GA) based, random waypoint distribution (RWD) and random target location (RTL) techniques. At last, as expected, the experimental results reveal that increased transmission ranges allow mobile sinks to track more pedestrians in theme parks.

\section{RELATED WORK}

In this section, we summarize the related previous studies of mobility models used in network simulations. Vukadinovic et al. [9] propose a simple framework to simulate mobility of theme park visitors. They use OpenStreetMap for the generation of the maps and calibrate the framework parameters according to the GPS traces. While their model is a trace-based mobility model of ordinary scenarios, we introduce a synthetic mobility model of the pedestrians for disaster scenarios in this paper. Aschenbruck et al. [10] model mobility of agents and disaster area for crowd behavior detection. They model obstacles, dangers, and shelters as separate zones and their disaster area is divided into various sub-areas such as incident site, casualties treatment area, transport zone, and hospital zone. An event- and role-based mobility model for disaster 
areas is proposed by Nelson et al. [11]. In their model, the movement patterns of people with different roles vary by their distinct reactions to disasters. For instance, a civilian aims to escape from a burning building while a firefigher runs into the burning building to save lives. While these studies explore different aspects of mobility modeling, none of them focuses on pedestrian mobility during disasters in large-scale areas without the use of vehicles.

\section{CONCLUSION}

In this paper, we presented the TP-D model with the purpose of evacuating the visitors from the theme parks during the disasters. The mobility of the pedestrians is modeled using real maps with the considerations of physical obstacles within the theme park and the social interactions among the visitors. We evaluated our model in comparison with TP, SLAW, RWP mobility models and real-world GPS traces.

As a future work, we plan to use the TP-D model as a baseline for testing the disaster response strategies. One possible strategy is using the smart-phones to build opportunistic social networks and broadcasting messages with critical information. Moreover, we believe that TP-D model can be adapted to various pedestrian mobility scenarios such as disasters in airports, state fairs and shopping malls.

\section{REFERENCES}

[1] "Theme index: Global attractions attendance report," Themed Entertainment Assoc. (TEA), Tech. Rep., 2013.

[2] V. Vukadinovic and S. Mangold, "Opportunistic wireless communication in theme parks: a study of visitors mobility," in Proc. ACM Workshop Challenged Netw., Sep. 2011, pp. 3-8.

[3] G. Solmaz and D. Turgut, "Optimizing event coverage in theme parks," Wireless Netw., vol. 20, no. 6, pp. 1445-1459, Aug. 2014.

[4] G. Solmaz, M. Akbas, and D. Turgut, "Modeling visitor movement in theme parks," in Proc. IEEE Local Comput. Netw., Oct. 2012, pp. 36-45.

[5] G. Solmaz and D. Turgut, "Theme park mobility in disaster scenarios," in Proc. IEEE Global Telecommun. Conf., Dec. 2013, pp. 377-382.

[6] M. Haklay and P. Weber, "OpenStreetMap: User-generated street maps." Pervasive Comput., vol. 7, no. 4, pp. 12-18, Dec. 2008.

[7] D. Helbing and A. Johansson, "Pedestrian, crowd and evacuation dynamics," Encyclopedia Complexity Syst. Sci., vol. 16, no. 4, pp. 6476-6495, 2010.

[8] K. Lee, S. Hong, S. J. Kim, I. Rhee, and S. Chong, "SLAW: self-similar least-action human walk," IEEE/ACM Trans. Netw., vol. 20, no. 2, pp. 515-529, Apr. 2012.

[9] V. Vukadinovic, F. Dreier, and S. Mangold, "A simple framework to simulate the mobility and activity of theme park visitors," in Proc. Winter Sim. Conf., Dec. 2011, pp. 3248-3260.

[10] N. Aschenbruck, E. Gerhards-Padilla, M. Gerharz, M. Frank, and P. Martini, "Modelling mobility in disaster area scenarios," in Proc. ACM Int. Conf. Modeling, Anal. and Sim. of Wireless and Mobile Syst., Oct. 2007, pp. 4-12.

[11] S. C. Nelson, A. F. Harris, III, and R. Kravets, "Event-driven, rolebased mobility in disaster recovery networks," in Proc. ACM Workshop Challenged Netw., Sep. 2007, pp. 27-34.

Gürkan Solmaz is currently working toward the $\mathrm{PhD}$ degree in Computer Science from the Department of Electrical Engineering and Computer Science, University of Central Florida (UCF). He received his MS degree in Computer Science from University of Central Florida and his BS degree in Computer Engineering from Middle East Technical University (METU), Turkey. His research interests include mobility modeling, mobile wireless sensor networks, and disaster resilience in networks. He is a student member of IEEE and ComSoc.
Damla Turgut is an Associate Professor at the Department of Electrical Engineering and Computer Science of University of Central Florida. She received her BS, MS, and PhD degrees from the Computer Science and Engineering Department of University of Texas at Arlington. Her research interests include wireless ad hoc, sensor, underwater and vehicular networks, as well as considerations of privacy in the Internet of Things. She is also interested in applying big data techniques for improving STEM education for women and minorities. Her recent honors and awards include being selected as an iSTEM Faculty Fellow for 2014-2015 and being featured in the UCF Women Making History series in March 2015. She was corecipient of the Best Paper Award at the IEEE ICC 2013. Dr. Turgut serves as a member of the editorial board and of the technical program committee of ACM and IEEE journals and international conferences. She is a member of IEEE, ACM, and the Upsilon Pi Epsilon honorary society. 